\title{
A Rare Case of a Penetrating Aortic Ulcer Due to Neisseria Gonorrhoeae
}

\author{
James Barr ${ }^{1}$, Richard G Bogle ${ }^{2,3^{*}}$, Arif Khokhar ${ }^{3}$, Arun Kumar Perikala ${ }^{3}$, Kathryn A Harris ${ }^{4}$, Rajan \\ Sharma ${ }^{2}$ and Robin Kanagasaby ${ }^{1}$ \\ ${ }^{1}$ Department of Cardiac Surgery, St George's University Hospitals NHS Foundation Trust, London, UK \\ ${ }^{2}$ Cardiology Clinical Academic Group, St George's University Hospitals NHS Foundation Trust, London, UK \\ ${ }^{3}$ Department of Cardiology, Epsom and St Helier University Hospitals NHS Trust, Surrey, UK \\ ${ }^{4}$ Department of Microbiology, Great Ormond Street Hospital for Children NHS Foundation Trust, London, \\ $\mathrm{UK}$
}

*Corresponding author: Richard G Bogle, Department of Cardiology, Epsom and St Helier University Hospitals NHS Trust, Wrythe Lane, Carshalton, Surrey SM5 1AA, UK, Tel: +44 07906439558; Fax: +44 02081817183; E-mail: richard.bogle1@nhs.net

Received Date: 30th August, 2017; Accepted Date: 8th October, 2017; Published Date: 23rd October, 2017

\begin{abstract}
Infectious aortitis is an extremely rare condition with an insidious presentation. Here we describe an unusual case of a penetrating ulcer in the ascending aorta that was caused by a chronic Neisseria gonorrhoea infection. The case illustrates the importance of taking a detailed sexual history of patients presenting with aortitis and the value of molecular microbiological techniques for establishing an accurate diagnosis.
\end{abstract}

\section{Keywords}

Neisseria Gonorrhoeae; Penetrating Aortic Ulcer

\section{Introduction}

Penetrating ulcers of the aorta are extremely uncommon and are usually associated with atherosclerotic vascular disease. Together with intramural haematoma and aortic dissection they compose the acute aortic syndromes [1]. These entities usually involve the disruption of the aortic media and present acutely with chest pain or haemodynamic instability [1].

Infective causes of aortic ulceration are rare in the modern era however it is well recognised that and certain bacteria have a predisposition for vascular infection [2]. Before the availability of penicillin infective aortic pathology was more common. Here we describe an unusual case of a penetrating aortic ulcer caused by Neisseria gonorrhoeae, a condition only reported once before and only confirmed following molecular microbiological analysis of aortic tissue removed at surgery.

\section{Case Report}

A 66 year old man was admitted to hospital with a 6 month history of increasing shortness of breath, lethargy and weight loss of about $20 \mathrm{~kg}$. Ten months previous he had experienced dysuria and transient swelling of his right knee which settled spontaneously without treatment and occurred following intercourse with multiple casual sexual partners whilst on holiday in Singapore. He did not seek medical attention at the time. Co-morbidity included type 2 diabetes mellitus and hypertension controlled with metformin and amlodipine respectively. He was divorced, had worked overseas and reported multiple casual sexual partners from around the world.

On examination he was pale, afebrile and had normal cardiovascular, respiratory and abdominal examination. There was no rash, lymphadenopathy or joint abnormalities. Investigations showed haemoglobin of $70 \mathrm{~g} / \mathrm{L}, \mathrm{MCV} 78$, RDW $17 \%$, WCC $32 \times 10^{9} / \mathrm{L}$ (neutrophils, 28), platelets 256 and albumin 25. Ferritin was 312 however the serum iron was 1.5 , transferrin 1.6 and transferrin saturation 4\%. C-Reactive Protein (CRP) was $160 \mathrm{mg} / \mathrm{L}$ and ESR $136 \mathrm{~mm} / \mathrm{h}$, immunoglobulins were normal, blood cultures sterile at 5 days of incubation and viral screen for hepatitis B, C, HIV-1 and 2 were negative. Treponemal antibody was negative. Autoantibodies and standard rheumatological screen were normal.

The initial working diagnosis was of possible abdominal or haematological malignancy because of the anaemia, weight loss and raised inflammatory markers. A contrast enhanced CT scan 
Citation: Barr J, Bogle RG, Khokhar A, Perikala AK, Harris KA, et al. (2017) A Rare Case of a Penetrating Aortic Ulcer Due to Neisseria Gonorrhoeae. J Clin Case Rep Case Stud 2017: 6-8. DOI : https://doi.org/10.29199/2637-9309/CCCS-101012.

of the chest, abdomen and pelvis was performed and showed a large global pericardial effusion with a dilated ascending aorta (50mm diameter) and was reported as a possible type A aortic dissection with probable intraluminal adherent thrombus. A transthoracic echocardiogram showed normal left ventricular size and function, tri-leaflet aortic valve with no aortic regurgitation and a moderate global pericardial effusion measuring $2 \mathrm{~cm}$ without tamponade physiology. An ECG gated CT of the aorta was performed and showed a contrast-filled out-pouching of the ascending aorta above the aortic root with the appearance of a penetrating ulcer. There was also an associated intraluminal filling defect in the ascending aorta attached to the wall.

The patient was taken to the operating room. Upon opening the pericardium $100 \mathrm{ml}$ of blood stained fluid was drained (Figure 1). The pericardium was thickened with diffuse inflammation. There was a penetrating ulcer located posteriorly on the ascending aorta at the level of the sino-tubular junction just superior to the left coronary ostium. There was diffuse aortic inflammation. Cardiopulmonary bypass was established with venous drainage from right atrium to distal ascending aorta and following aortic cross clamping and cardioplegia the diseased part of the ascending aorta was excised and replaced with a $28 \mathrm{~mm}$ Hemashield interposition vascular graft. The patient was rewarmed and separated from cardiopulmonary bypass without inotropic support. He recovered well from his surgery and was discharged home 7 days later.

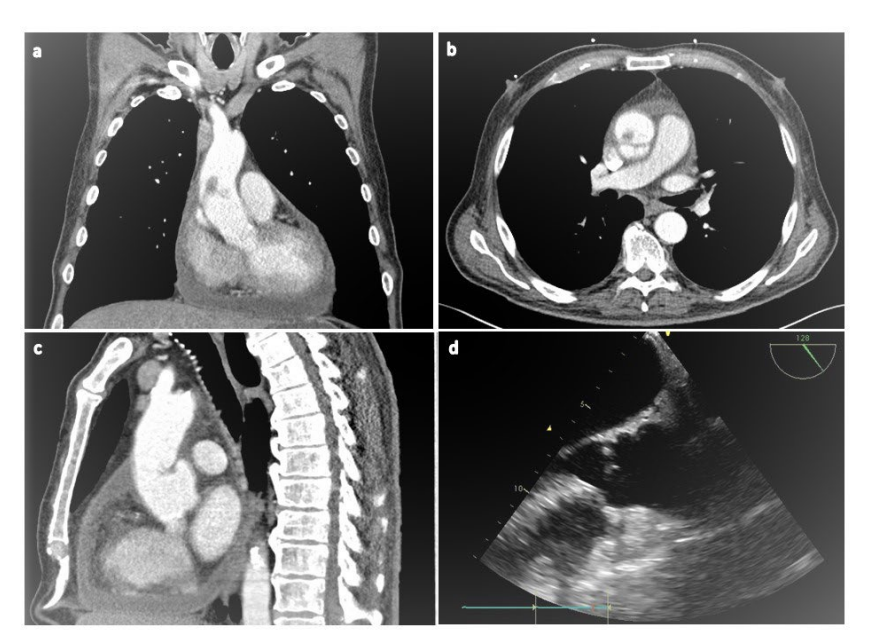

Figure 1 (a-c): Coronal, transverse and sagittal slices from ECG-gated CT scan demonstrating an enhancing bulge at the sino-tubular junction suggestive of penetrating ulcer; (d): Intra-operative Trans-oesophageal echocardiography of ascending aorta demonstrating a dilated ascending aorta with interruption of the vascular integrity.

Cytology of pericardial fluid showed lymphocytic effusion and staining of the aortic tissue for micro-organisms and fungi was negative. Histological analysis of the aorta showed patchy inflammation within the adventitia, but no extension of inflammation into the media. Material from the inside of the aorta comprised fibrino-inflammatory material with a nodular appearance, in parts, but no significant atherosclerosis was detected. The tissue sample was sent for molecular diagnostic analysis to the Clinical Microbiology Laboratory at Great Ormond Street Hospital. This laboratory has 15 -years of experience in performing broad-range $16 \mathrm{~S}$ rDNA PCR as part of the routine investigation of endocardial and vascular specimens and the methodology is able to identify most bacterial to species level, including $N$. gonorrhoea and, all bacteria to genus level $[3,4]$. The Polymerase Chain Reaction (PCR) for the 16S rRNA gene was positive and the amplicon was sequenced and identified as $N$. gonorrhoeae by BLAST analysis against the GenBank database (https://blast.ncbi.nlm.nih.gov). Following discussion in our endocarditis multidisciplinary meeting the patient was readmitted for a course of intravenous Ceftriaxone $2 \mathrm{~g}$ once daily for a period of 4 weeks. A specialist genitourinary medicine assessment showed no evidence of genital gonorrhoeae and the patient remains well and has completed a full course of treatment

\section{Discussion}

Bacterial infections of the aorta are extremely rare. This is hypothesised to be due to the resistance of the aortic intima to bacterial adhesion and migration. Pathology such as atherosclerosis or cystic medial necrosis predispose damage the endothelium and promote adherence and bacterial grown especially if associated with immunosuppressive conditions such as diabetes. Organisms such as $N$. gonorrhoeae are associated with vascular infection and in particular, of the large vessels such as the aorta. Infection occurs in the context of Disseminate Gonococcal Infection (DGI) either by direct seeding to an area of damaged intima due to atherosclerosis, or by haematogenous spread to the vessel wall through the vasa vasorum. We believe in this case that haematogenous spread was the most likely mechanism as there was no evidence of atherosclerotic disease on histology although endothelial dysfunction secondary to diabetes and hypertension may have resulted in increased susceptibility to bacterial invasion due to loss of protective factors such as nitric oxide.

Patients with infective aortitis usually present with a sub-acute illness as was the case in our patient. At presentation, the patient had no localising symptoms or signs and there was a wide differential diagnosis. The salient pathology was detected only after detailed cross-sectional imaging. Anaemia and markedly raised CRP and ESR resulted from the chronic infection/inflammation but were non-specific findings.

Gonorrhoea is an ancient disease colloquially known as the "clap", a name derived from Les Clapiers, the Parisian district where prostitutes were housed [5]. It remains a major 21 st century international health problem with a worldwide incidence rising from 62 million cases in 1995 to 106 million cases in 2008 [5]. N. gonorrhoeae strains acquire resistance to all classes of antimicrobial agents [6]. N. gonorrhoeae only is a selective infection of humans and usually involves the genital mucosal surfaces leading to cervicitis in women and urethritis in men. In $0.5-3 \%$ of cases systemic spread results in DGI [7]. This results in a tenosynovitis-arthritis syndrome accompanied by systemic manifestations or an isolated suppurative arthritis usually involving a single large joint [8]. DGI most likely explained the patient's transient dysuria and monoarthritis of the knee that occurred 10 months prior to his presentation. Involvement of the heart or vascular system is extremely rare. Endocarditis is reported and tends to affect the aortic valve and can be complicated by aortic root abscess, large sized vegetations and this is infection is associated with a high 
Citation: Barr J, Bogle RG, Khokhar A, Perikala AK, Harris KA, et al. (2017) A Rare Case of a Penetrating Aortic Ulcer Due to Neisseria Gonorrhoeae. J Clin Case Rep Case Stud 2017: 6-8.

mortality and relapse/recurrence [9,10]. The disease usually affects younger, sexually active people from lower socioeconomic classes. Cases of myocarditis have also been reported and usually symptoms resolve with treatment of gonorrhoea [11,12].

Other cases reports of gonorrhoea affecting the aorta have also been published with larger cases series from the pre-antibiotic era $[13,14]$ with only a few reported in the 21 st century [15-17]. Cases of penetrating ulcer caused by N. gonorrhoea are extremely rare. In 2011 a case of a patient with a bicuspid aortic valve and aneurysm of the ascending aorta presenting with fever and chest pain was found to have $N$. gonorrhoeae isolated from an intraluminal thrombus adherent to the ulcer but not directly from the aortic wall [18]. We now report a case of a penetrating aortic ulcer caused by $N$. gonorrhoeae arising from the adventitia and associated with intraluminal thrombus. Our case illustrates two important lessons. First the importance of taking a detailed sexual history from a patient presenting with an aortic ulcer and specifically enquiring about large joint and genital symptoms. Second, the importance in the use of modern molecular biology techniques such as broad-range 16S rRNA gene PCR and amplicon sequencing in the diagnosis of this infection since traditional microscopy, bacterial staining and cultures were negative possibly due to the fastidious nature of $N$. gonorrhoeae or due antibiotic treatment given at the time of surgery.

\section{Conclusion}

$N$. gonorrhoeae is a sexually transmitted infection with increasing incidence and resistance to antimicrobial therapy. DGI is rare and involvement of the heart and ascending aorta is rarer still. We report a rare case of a penetrating aortic ulcer caused by $N$. gonorrhoeae and described the management of the condition.

\section{References}

1. Mussa FF, Horton JD, Moridzadeh R, Nicholson J, Trimarchi S, et al (2016) Acute Aortic Dissection and Intramural Hematoma: A Systematic Review. JAMA 316: 754-763.

2. Lopes RJ, Almeida J, Dias PJ, Pinho P, Maciel MJ (2009) Infectious thoracic aortitis: a literature review. Clin Cardiol 32: 488-490.

3. Harris KA, Hartley J (2003) Development of a broad-range $16 \mathrm{~S}$ rDNA $\mathrm{PCR}$ for use in the routine diagnostic clinical microbiological service. J Med Microbiol 52: 685-691.

4. Harris KA, Yam T, Jalili S, Williams OM, Alshafi K, et al. (2014) Service evaluation to establish the sensitivity, specificity and additional value of broad range 16S rDNA PCR for the diagnosis of infective endocarditis from resected endocardial material in patients from eight UK and Ireland hospitals. Eur J Clin Microbiol Infect Dis 33: 2061-2066.

5. World Health Organization, Department of Reproductive Health and Research (2012) Global incidence and prevalence of selected curable sexually transmitted infections - 2008. World Health Organization, Geneva, Switzerland. Pg no: 20.

6. Unemo M, Shafer WM (2014) Antimicrobial resistance in Neisseria gonorrhoeae in the 21st century: past, evolution, and future. Clin Microbiol Rev 27: 587-613.

7. Lohani S, Nazir S, Tachamo N, Patel N (2016) Disseminated gonococcal infection: an unusual presentation. J Community Hosp Intern Med Perspect 6: 31841.

8. Rice PA(2005) Gonococcal arthritis (disseminated gonococcal infection). Infect Dis Clin North Am 19: 853-961.

9. Jackman JD, Glamann DB (1991) Gonococcal endocarditis: twenty-five year experience. Am J Med Sci 301: 221-230.

10. de Campos FP, Kawabata VS, Bittencourt MS, Lovisolo SM, Felipe-Silva A, et al. (2016) Gonococcal endocarditis: an ever-present threat. Autops Case Rep 6: 19-25.

11. Bunker D, Kerr LD (2015) Acute Myopericarditis Likely Secondary to Disseminated Gonococcal Infection. Case Rep Infect Dis 2015: doi: $10.1155 / 2015 / 385126$

12. Fraser HS, Liburd AL, Figueroa JP, Nicholson GA, James OB, et al. (1974) Gonococcaemia with arthritis, dermatitis and myocarditis. Postgrad Med J 50: 759-764.

13. Currens, JH, Faulkner JM (1943) Gonococcal mycotic aneurysm of the aorta: report of a case superimposed upon a syphilitic aorta. Ann Intern Med 19: 155-162.

14. Parkhurst GF, Dekcer JP (1955) Bacterial aortitis and mycotic aneurysm of the aorta; a report of twelve cases. Am J Pathol 31: 821-835.

15. Markowicz S, Anstey JR, Hites M, Montesinos I, Roisin S, et al. (2014) Gonococcal aneurysm of the ascending aorta: case report and review of Neisseria gonorrhoeae endovascular infections. Sex Transm Dis 41: 111-113.

16. Pätilä T, Kurki T, Ihlberg L (2012) Isolated gonococcal ascending aorta aneurysm. Interact Cardiovasc Thorac Surg 15: 183-185.

17. Oumeiri BE, Eynden FV, Stefanidis C, Antoine M, Nooten GV (2015) Gonococcal ascending aortic aneurysm with penetrating ulcer and bovine arch. Asian Cardiovasc Thorac Ann 23: 861-863.

18. Woo JS, Rabkin DG, Mokadam NA, Rendi MH, Aldea GS (2011) Gonococcal ascending aortitis with penetrating ulcers and intraluminal thrombus. Ann Thorac Surg 91: 910-912. 